\title{
Isomorphisms of subcategories of fusion systems of blocks and Clifford theory
}

\author{
Morton E. Harris \\ Communicated by Britta Spaeth
}

\begin{abstract}
Let $k$ be an algebraically closed field of prime characteristic $p$. Let $G$ be a finite group, let $N$ be a normal subgroup of $G$, and let $c$ be a $G$-stable block of $k N$ so that $(k N) c$ is a $p$-permutation $G$-algebra. As in Section 8.6 of [M. Linckelmann, The Block Theory of finite Group Algebras: Volume 2, London Math. Soc. Stud. Texts 92, Cambridge University, Cambridge, 2018], a $(G, N, c)$-Brauer pair $\left(R, f_{R}\right)$ consists of a $p$-subgroup $R$ of $G$ and a block $f_{R}$ of $\left(k C_{N}(R)\right)$. If $Q$ is a defect group of $c$ and $f_{Q} \in B \ell\left(k C_{N}(Q)\right)$, then $\left(Q, f_{Q}\right)$ is a $(G, N, c)$-Brauer pair. The $(G, N, c)$-Brauer pairs form a (finite) poset. Set $H=N_{G}\left(Q, f_{Q}\right)$ so that $\left(Q, f_{Q}\right)$ is an $\left(H, C_{N}(Q), f_{Q}\right)$-Brauer pair. We extend Lemma 8.6.4 of the above book to show that if $\left(U, f_{U}\right)$ is a maximal $(G, N, c)$-Brauer pair containing $\left(Q, f_{Q}\right)$, then $\left(U, f_{U}\right)$ is a maximal $\left(H, C_{N}(c), f_{Q}\right)$ Brauer pair containing $\left(Q, f_{Q}\right)$ and conversely. Our main result shows that the subcategories of $\mathcal{F}_{\left(U, f_{U}\right)}(G, N, c)$ and $\mathcal{F}_{\left(U, f_{U}\right)}\left(H, C_{N}(Q), f_{Q}\right)$ of objects between and including $\left(Q, f_{Q}\right)$ and $\left(U, f_{U}\right)$ are isomorphic. We close with an application to the Clifford theory of blocks.
\end{abstract}

Our notation and terminology are standard and tend to follow the notation and terminology of [2-4].

Let $k$ be an algebraically closed field of prime characteristic $p$. Let $G$ be a finite group, let $N \unlhd G$, and let $c \in B \ell(k N)^{G}$. Thus we are in the situation of fusion systems of blocks and Clifford theory of [3, Section 8.6]. This section is devoted to a study of fusion systems arising from the $G$-algebra $(k N) c$ and the results of [2, Section 5.9].

Thus, by [3, Definition 8.6.1], a $(G, N, c)$-Brauer pair is a pair $\left(R, f_{R}\right)$, where $R$ is a $p$-subgroup of $G$ and $f_{R} \in B \ell\left(k C_{N}(R) B r_{R}(c)\right)$. The results of [3, Section 8.6] imply that the set of $(G, N, c)$-Brauer pairs is a (finite) poset under a defined inclusion. Moreover, by [3, Definition 8.6.2 and Theorem 8.6.3], if $\left(P, f_{P}\right)$ is a maximal $(G, N, c)$-Brauer pair, then $\mathcal{F}_{\left(P, f_{P}\right)}(G, N, c)$ is a fusion system on $P$ and $(P N) / N \in S y l_{p}(G / N)$. Note that all maximal $(G, N, c)$-Brauer pairs are $G$-conjugate.

Let $Q$ be a defect group of $c \in B \ell(k N)$. Thus $B r(c) \in B \ell\left(k N_{N}(Q)\right)$ with defect group $Q$ and, [4, Theorem 5.5.15], $B r_{Q}(c)$ corresponds to an orbit $\Gamma$ of $N_{H}(Q)$ on $B \ell\left(k C_{N}(Q)\right)$ such that $\Gamma$ is the set of blocks of $k C_{N}(Q)$ that are 
covered by $B \ell_{Q}(c)$ and $B r_{Q}(c)=\sum_{\gamma \in \Gamma} \gamma$ is an orthogonal sum of blocks of $k C_{N}(Q)$. Thus $\{(Q, \gamma) \mid \gamma \in \Gamma\}$ is the set of $(G, N, c)$-Brauer pairs with first component $Q$. Let $f_{Q} \in \Gamma$.

Let $\left(R, f_{R}\right)$ be a maximal $(G, N, c)$-Brauer pair. Then, as in [3, Section 8.6], $R \cap N$ is a defect group of $c \in B \ell(k N)^{G}$. Then there is an $x \in N$ such that ${ }^{x}\left(R, f_{R}\right)=\left({ }^{x} R,{ }^{x} f_{R}\right)$ is maximal $(G, N, c)$-Brauer pair that contains $\left(Q, f_{Q}\right)$.

Let $\left(P, f_{P}\right)$ be a maximal $(G, N, c)$-Brauer pair that contains $\left(Q, f_{Q}\right)$. Thus $P \cap N=Q$ and $Q \unlhd P \unlhd N_{G}(P) \leq N_{G}(Q)$.

Set

$$
H=N_{G}\left(Q, f_{Q}\right)
$$

so that $Q \unlhd H, C_{N}(Q) \unlhd H$ and $\left(Q, f_{Q}\right)$ is an $\left(H, C_{N}(Q), f_{Q}\right)$-Brauer pair. Thus if $\left(U, f_{U}\right)$ is a maximal $\left(H, C_{N}(Q), f_{Q}\right)$-Brauer pair that contains $\left(Q, f_{Q}\right)$, then $\mathcal{F}_{\left(U, f_{U}\right)}\left(H, C_{N}(Q), f_{Q}\right)$ is a fusion system such that

$$
\left(U C_{N}(Q)\right) / C_{N}(Q) \in \operatorname{Syl}_{p}\left(H / C_{N}(Q)\right) .
$$

Set

$$
\begin{aligned}
\Phi\left(G, N, c \mid\left(Q, f_{Q}\right)\right)=\left\{\left(R, f_{R}\right) \mid\right. & \left(R, f_{R}\right) \text { is a }(G, N, c) \text {-Brauer pair } \\
& \text { such that }\left(Q, f_{Q}\right) \leq\left(R, f_{R}\right) \\
& \text { as }(G, N, c) \text {-Brauer pairs }\}
\end{aligned}
$$

Clearly, $\left(Q, f_{Q}\right) \in \Phi\left(G, N, c \mid\left(Q, f_{Q}\right)\right)$.

Similarly, $\Phi\left(H, C_{N}(Q), f_{Q} \mid\left(Q, f_{Q}\right)\right)$ is defined and

$$
\left(Q, f_{Q}\right) \in \Phi\left(H, C_{N}(c), f_{Q} \mid\left(Q, f_{Q}\right)\right) .
$$

Remark 1. If $\left(R, f_{R}\right) \in \Phi\left(G, N, c \mid\left(Q, f_{Q}\right)\right)$, then we may choose a maximal $(G, N, c)$-Brauer pair containing $\left(R, f_{R}\right)$ so that $R \cap N=Q$. Thus

$$
Q \unlhd R \leq N_{G}(R) \leq N_{G}(Q) .
$$

In fact, $N_{G}\left(R, f_{R}\right) \leq N_{G}\left(Q, f_{Q}\right)$ by [2, Proposition 5.9.9].

A similar result holds for $\Phi\left(H, C_{N}(Q), f_{Q} \mid\left(Q, f_{Q}\right)\right)$.

As suggested by [3, Lemma 8.6.4]:

Lemma 2. Let $S$ be a p-subgroup of $G$ such that $Q \leq S$, and let $e \in B \ell\left(k C_{N}(S)\right)$. The following two conditions are equivalent:

(a) $(S, e) \in \Phi\left(G, N, c \mid\left(Q, f_{Q}\right)\right)$;

(b) $(S, e) \in \Phi\left(H, C_{N}(Q), f_{Q} \mid\left(Q, f_{Q}\right)\right)$. 
Proof. Assume (a). Then $Q \unlhd S$, and $f_{Q}$ is the unique $S$-stable block of $k C_{N}(Q)$ such that $B r_{S}\left(f_{Q}\right) e=e$. Since $C_{N}(S)=C_{C_{N}(Q)}(S)$, (b) holds. The proof of the converse is similar.

Let $S, T$ be $p$-subgroups of $G$ such that $Q \leq S \leq T$, and let $f_{S} \in B \ell\left(k C_{N}(S)\right)$ and $f_{T} \in B \ell\left(k C_{N}(T)\right)$ be such that

$$
\begin{aligned}
& \left(S, f_{S}\right) \in \Phi\left(G, N, c \mid\left(Q, f_{Q}\right)\right), \\
& \left(T, f_{T}\right) \in \Phi\left(G, N, c \mid\left(Q, f_{Q}\right)\right) .
\end{aligned}
$$

Thus

$$
\begin{aligned}
& \left(S, f_{S}\right) \in \Phi\left(H, C_{N}(Q), f_{Q} \mid\left(Q, f_{Q}\right)\right), \\
& \left(T, f_{T}\right) \in \Phi\left(H, C_{N}(Q), f_{Q} \mid\left(Q, f_{Q}\right)\right) .
\end{aligned}
$$

Lemma 3. The following two conditions are equivalent:

(a) $\left(S, f_{S}\right) \leq\left(T, f_{T}\right)$ as $(G, N, c)$-Brauer pairs;

(b) $\left(S, f_{S}\right) \leq\left(T, f_{T}\right)$ as $\left(H, C_{N}(Q), f_{Q}\right)$-Brauer pairs.

Proof. Clearly, we may assume that $Q<S<T$. We proceed by induction on $|T: Q|$. Thus we may assume that $T$ is a minimal subgroup of $H$ such that there is a subgroup $S$ of $T$ and a $(G, N, c)$-Brauer pair $\left(S, f_{S}\right)$ such that (a) and (b) are not equivalent for $\left(S, f_{S}\right)$ and $\left(T, f_{T}\right)$.

Clearly, there is a subgroup $R$ of $T$ such that $Q<S \leq R \unlhd T$ with $R<T$. Let $f_{R} \in B \ell\left(k C_{N}(R)\right)$ be the unique $(G, N, c)$-Brauer pair so that $\left(R, f_{R}\right) \leq\left(T, f_{T}\right)$ as $(G, N, c)$-Brauer pairs. Thus $f_{R}$ is the unique $T$-stable block of $k C_{N}(R)$ such that $B_{T}\left(f_{R}\right) f_{T}=f_{T}$. Hence $\left(R, f_{R}\right)$ is an $\left(H, C_{N}(Q), f_{Q}\right)$-Brauer pair such that $\left(R, f_{R}\right) \leq\left(T, f_{T}\right)$ as $\left(H, C_{N}(Q), f_{Q}\right)$-Brauer pairs.

Assume (a). Thus $\left(S, f_{S}\right) \leq\left(R, f_{R}\right)$ as $(G, N, c)$-Brauer pairs. Since we have $|R: Q|<|T: Q|$, we conclude that $\left(S, f_{S}\right) \leq\left(R, f_{R}\right)$ as $\left(H, C_{N}(Q), f_{Q}\right)$-Brauer pairs. Thus (b) holds.

Assume (b). Then the same argument shows that (a) holds. This contradiction establishes the lemma.

Thus we have:

Corollary 4. Let $\left(U, f_{U}\right) \in \Phi\left(G, N, c \mid\left(Q, f_{Q}\right)\right.$ so that

$$
\left.\left(U, f_{U}\right) \in \Phi\left(H, C_{N}(Q), f_{Q}\right) \mid\left(Q, f_{Q}\right)\right) .
$$

The following two condition are equivalent:

(a) $\left(U, f_{U}\right)$ is a maximal $(G, N, c)$-Brauer pair;

(b) $\left(U, f_{U}\right)$ is a maximal $\left(H, C_{N}(Q), f_{Q}\right)$-Brauer pair. 
Let $\left(U, f_{U}\right)$ satisfy (a) and (b) of Corollary 4 . Then $\mathcal{F}_{\left(U, f_{U}\right)}(G, N, c)$ and $\mathcal{F}_{\left(U, f_{U}\right)}\left(H, C_{N}(Q), f_{Q}\right)$ are fusion systems by [3, Theorem 8.6.3].

Proposition 5. Let

$$
\left(S, f_{S}\right) \in \Phi\left(G, N, c \mid\left(Q, f_{Q}\right)\right)
$$

be such that $\left(S, f_{S}\right) \leq\left(U, f_{U}\right)$ as $(G, N,, c)$-Brauer pairs. Also let $\left(T, f_{T}\right)$ be a $(G, N, c)$-Brauer pair such that $\left(T, f_{T}\right) \leq\left(U, f_{U}\right)$ as $(G, N, c)$-Brauer pairs, and let $x \in G$ be such that ${ }^{x}\left(S, f_{S}\right) \leq\left(T, f_{T}\right)$ as $(G, N, c)$-Brauer pairs. Then

(a) $x \in H$ and $\left(T, f_{T}\right) \in \Phi\left(G, N, c \mid\left(Q, f_{Q}\right)\right)$;

(b) ${ }^{x}\left(S, f_{S}\right) \leq\left(T, f_{T}\right)$ as $\left(H, C_{N}(Q), f_{Q}\right)$-Brauer pairs.

Proof. Here ${ }^{x}\left(Q, f_{Q}\right) \leq\left(T, f_{T}\right) \leq\left(U, f_{U}\right)$ and $\left(Q, f_{Q}\right) \leq\left(U, f_{U}\right)$. Since we have ${ }^{x} Q \leq U \cap N=Q$, both (a) and (b) hold.

Our main result is: Let $\mathcal{C}$ denote the full subcategory of $\mathcal{F}_{\left(U, f_{U}\right)}(G, N, c)$ of objects in $\Phi\left(G, N, c \mid\left(Q, f_{Q}\right)\right)$, and let $\mathscr{D}$ denote the full subcategory of

$$
\mathcal{F}_{\left(U, f_{U}\right)}\left(H, C_{N}(Q), f_{Q} \mid\left(Q, f_{Q}\right)\right)
$$

of objects in $\Phi\left(H, C_{N}(Q), f_{Q} \mid\left(Q, f_{Q}\right)\right)$. Thus we have:

Theorem 6. The identity functor $I: D \rightarrow \mathcal{D}$ is an isomorphism of categories.

Our last results relate to blocks.

Lemma 7. Let $B \in B \ell((k G) c)$ so that $B c=B$. Then there is a defect group $P$ of $B$ such that $P \cap N=Q$ and a block $f_{P}$ of $k C_{N}(P)$ such that

$$
\left(P, f_{P}\right) \in \Phi\left(G, N, c \mid\left(Q, f_{Q}\right)\right) .
$$

Proof. We may choose a defect group $R$ of $B$ such that $R \cap N=Q$. Hence $N_{G}(R) \leq N_{G}(Q), C_{G}(R) \leq C_{G}(Q)$ and $C_{N}(R) \leq C_{N}(Q)$. Here we have that $\operatorname{Br}_{R}(B) \in B \ell\left(k N_{G}(R)\right)$ has defect group $R$ and $C_{N}(R) \unlhd N_{G}(R)$. Thus there is an orbit $\Delta$ of $N_{G}(R)$ on $B \ell\left(k C_{N}(R)\right)$ such that $\Delta$ consists of the blocks of $k C_{N}(R)$ that are covered by $B_{R}(B)$ and

$$
B r_{R}(B)=B r_{R}(B)\left(\sum_{\delta \in \Delta} \delta\right)=B r_{R}(B) B_{R}(c)=B_{R}(B)\left(\sum_{\delta \in \Delta} \delta\right)\left(\sum_{\gamma \in \Gamma} B_{R}(\gamma)\right) .
$$

Thus there is an $x \in N_{N}(Q)$ and a $\delta \in \Delta$ so that $B r_{x}(B)\left({ }^{x} \delta\right) B r_{x}\left(f_{Q}\right) \neq 0$. Here ${ }^{x} R$ is a defect group of $B$,

$$
\left({ }^{x} R\right) \cap N=Q, \quad{ }^{x} \delta \in B \ell\left(k C_{N}\left({ }^{x} R\right)\right) \quad \text { and } \quad\left({ }^{x} \delta\right) B r x_{R}\left(f_{Q}\right) \neq 0 .
$$


Since $f_{Q} \in B \ell\left(k C_{N}(Q)\right)$ and $C_{N}\left({ }^{x} R\right) \leq C_{N}(Q),\left({ }^{x} \delta\right) B r_{x} R\left(f_{Q}\right)={ }^{x} \delta$. Since ${ }^{x} R$ fixes $B r_{Q}(c)$, it permutes $\Gamma$. Thus, since $B r^{x} R\left(f_{Q}\right) \neq 0,{ }^{x} R$ fixes $f_{Q}$. Thus $\left({ }^{x} R,{ }^{x} \delta\right)$ is a $(G, N, c)$-Brauer pair, $\left(Q, f_{Q}\right) \leq\left({ }^{x} R,{ }^{x} \delta\right)$ and ${ }^{x} R$ is a defect group of $B$. The proof is complete.

Let $B \in B \ell((k G) c)$. By [1, Theorem], there is a unique

$$
\beta \in B \ell\left(k N_{G}(Q) B r_{Q}(c)\right) \text { such that } \beta^{G}=B .
$$

Here $\beta B r_{Q}(c)=\beta$ and $\operatorname{Br}_{Q}(c)=\sum_{\gamma \in \Gamma} \gamma$. Since $C_{N}(Q) \unlhd N_{G}(Q)$, we have $\Gamma \subseteq B \ell\left(k C_{N}(Q)\right)$, and so $\beta$ covers $f_{Q}$. Since $H=N_{G}\left(Q, f_{Q}\right)$, there is a unique $b \in B \ell\left((k H) f_{Q}\right)$ such that $\operatorname{Tr}_{H}^{N_{G}(Q)}(b)=\beta$. Hence $\left(\operatorname{Tr}_{H}^{N_{G}}(Q)(b)\right){ }^{G}=B$.

Here, by [4, Corollary 5.5.6], we have $f_{Q} \in B \ell\left(k Q C_{N}(Q)\right)^{H}$. Thus $b$ is an $\left(H, Q C_{N}(Q), f_{Q}\right)$-Brauer pair since $b f_{Q}=b$. We assert that $Q$ is a defect group of $f_{Q} \in B \ell\left(k Q C_{N}(Q)\right)$. Indeed, $B r_{Q}(c) \in B \ell\left(k N_{N}(Q)\right)$ with defect group $Q$. Here $B r_{Q}(c)=\sum_{\gamma \in \Gamma} \gamma$ is an orthogonal decomposition of blocks of $k C_{N}(Q)$ such that $f_{Q} \in \Gamma$. Also if $\gamma \in \Gamma$, then $\gamma \in B \ell\left(k Q C_{N}(Q)\right)$ by [4, Corollary 5.5.6] and $Q C_{N}(Q) \unlhd N_{N}(Q)$. Thus $B r_{Q}(c)$ covers $\gamma$ for any $\gamma \in \Gamma$. Hence we have that $Q \cap\left(Q C_{N}(Q)\right)=Q$ is a defect group of $\gamma$ for any $\gamma \in \Gamma$. Thus $Q$ is a defect group of $f_{Q} \in B \ell\left(k Q C_{N}(Q)\right)$, where $Q C_{N}(Q) \unlhd H, f_{Q} \in B \ell\left(k Q C_{N}(Q)\right)^{H}$.

By Lemma 7, there is a defect group $D$ of $b$ such that $D \cap Q C_{N}(Q)=Q$ and a block $f_{D}$ of $C_{Q C_{N}((Q)}(D)$ such that $\left(Q, f_{Q}\right) \leq\left(D, f_{D}\right)$ as $\left(H, Q C_{N}(Q), f_{Q}\right)$ Brauer pairs. Here $C_{Q C_{N}(Q)}(D) \leq C_{Q C_{N}(Q)}(Q)=C_{N}(Q)$, and so

$$
C_{Q C_{N}(Q)}(D)=C_{C_{N}(Q)}(D)=C_{N}(D) .
$$

Thus $B r_{D}\left(f_{Q}\right) f_{D}=f_{D}$ and $\left(D, f_{D}\right)$ is an $\left(H, C_{N}(Q), f_{Q}\right)$-Brauer pair such that $\left(Q, f_{Q}\right) \leq\left(D, f_{D}\right)$ as $\left(H, C_{N}(Q), f_{Q}\right)$-Brauer pairs.

Here $D$ is a defect group of $b$ and $B$. Let $\mathcal{C}\left(D, f_{D}\right)$ denote the full subcategory of $\mathcal{C}$ of objects $\left(S, f_{S}\right)$ of $\mathcal{C}$ such that $\left(S, f_{S}\right) \leq\left(D, f_{D}\right)$ as $(G, N, c)$-Brauer pairs, and let $\mathscr{D}\left(D, f_{D}\right)$ denote the full subcategory of $\mathscr{D}$ of objects $\left(T, f_{T}\right)$ of $\mathscr{D}$ such that $\left(T, f_{T}\right) \leq\left(D, f_{D}\right)$ as $\left(H, C_{N}(Q), f_{Q}\right)$-Brauer pairs.

Recall that $D$ is a defect group of $b$ and $B$. Thus, by choosing a maximal $\left(H, C_{N}(Q), f_{Q}\right)$-Brauer pair $\left(U, f_{U}\right)$ such that

$$
\left.\left(D,, f_{D}\right) \leq U, f_{U}\right)
$$

as $\left(H, C_{N}(Q), f_{Q}\right)$-Brauer pairs and applying Theorem 6, we conclude:

Corollary 8. The identity functor $I: D\left(D, f_{D}\right) \rightarrow \mathcal{C}\left(D, f_{D}\right)$ is an isomorphism of categories. 


\section{Bibliography}

[1] M. E. Harris and R. Knörr, Brauer correspondence for covering blocks of finite groups, Comm. Algebra 13 (1985), 1213-1218.

[2] M. Linckelmann, The Block Theory of Finite Group Algebras: Volume 1, London Math. Soc. Stud. Texts 91, Cambridge University, Cambridge, 2018.

[3] M. Linckelmann, The Block Theory of finite Group Algebras: Volume 2, London Math. Soc. Stud. Texts 92, Cambridge University, Cambridge, 2018.

[4] H. Nagao and Y. Tsushima, Representations of Finite Groups, Academic Press, San Diego, 1989.

Received October 25, 2019; revised February 19, 2020.

\section{Author information}

Morton E. Harris, Department of Mathematics, Statistics, and Computer Science (M/C 249), University of Illinois at Chicago, 851 South Morgan Street, Chicago, IL60607-7045, USA.

E-mail: meharris@uic . edu 